\title{
Association between polymorphisms in EGFR and tumor response during cetuximab and oxaliplatin-based combination therapy in metastatic colorectal cancer: Analysis of data from two clinical trials
}

\author{
HIROMICHI MAEDA $^{1 *}$, SHOICHI HAZAMA ${ }^{2,3^{*}}$, SHIGEYOSHI IWAMOTO ${ }^{4}$, KOJI OBA $^{5}$, RYOUICHI TSUNEDOMI $^{2}$, \\ NAOKO OKAYAMA $^{6}$, YUTAKA SUEHIRO ${ }^{7}$, TAKAHIRO YAMASAKI ${ }^{7}$, YUKI NAKAGAMI ${ }^{2,3}$, NOBUAKI SUZUKI ${ }^{2}$, \\ HIROAKI NAGANO $^{2}$, JUNICHI SAKAMOTO ${ }^{8}$, HIDEYUKI MISHIMA ${ }^{4}$ and NAOKI NAGATA ${ }^{9}$ \\ ${ }^{1}$ Cancer Treatment Center, Kochi Medical School Hospital, Kochi University, Nankoku, Kochi 783-8505; \\ ${ }^{2}$ Department of Gastroenterological, Breast and Endocrine Surgery, Yamaguchi University Graduate School \\ of Medicine; ${ }^{3}$ Department of Translational Research and Developmental Therapeutics Against Cancer, \\ Yamaguchi University School of Medicine, Ube, Yamaguchi 755-8505; ${ }^{4}$ Cancer Center, Aichi Medical University, \\ Nagakute, Aichi 480-1195; ${ }^{5}$ Department of Biostatistics, Graduate School of Medicine, University of Tokyo, \\ Bunkyo, Tokyo 113-0033; ${ }^{6}$ Division of Laboratory, Yamaguchi University Hospital; \\ ${ }^{7}$ Department of Oncology and Laboratory Medicine, Yamaguchi University Graduate School of Medicine, \\ Ube, Yamaguchi 755-8505; ${ }^{8}$ Tokai Central Hospital, Kakamigahara, Gifu 504-8601; \\ ${ }^{9}$ Kitakyushu General Hospital, Kitakyushu, Fukuoka 802-8517, Japan
}

Received December 17, 2018; Accepted July 17, 2019

DOI: $10.3892 / 01.2019 .10787$

\begin{abstract}
Predicting tumor response prior to starting anti-epidermal growth factor receptor (EGFR) antibody therapy would benefit patients with advanced/metastatic colorectal cancer (mCRC). The present study investigated the association between efficacy of cetuximab treatment and gene polymorphisms of fragment $\mathrm{C} \gamma$ receptor $(\mathrm{Fc} \gamma \mathrm{R}) 2 \mathrm{~A}, \mathrm{Fc} \gamma \mathrm{R} 3 \mathrm{~A}$ and EGFR in patients with extended RAS/BRAF wild-type mCRC. Clinical data and specimens were obtained from 90 patients who participated in either of two clinical studies evaluating the first-line, cetuximab plus oxaliplatin-based treatment. It was hypothesized that polymorphisms $\mathrm{H} / \mathrm{H}$ of Fc $\gamma R 2 A$, V/V of Fc $\gamma R 3 A$, K/K of EGFR and <36 CA repeats in the EGFR gene may be associated with a favorable tumor response. Multivariate analysis demonstrated that patients with the $\mathrm{H} / \mathrm{H}$ polymorphism tended to have an improved tumor response compared with the non-H/H population, although the result was not significant [odds ratio, 2.25;
\end{abstract}

Correspondence to: Dr Hiromichi Maeda, Cancer Treatment Center, Kochi Medical School Hospital, Kochi University, 185-1 Kohasu, Oko-cho, Nankoku, Kochi 783-8505, Japan

E-mail: hmaeda@kochi-u.ac.jp

${ }^{*}$ Contributed equally

Key words: cetuximab, polymorphism, oxaliplatin, epidermal growth factor receptor, fragment $\mathrm{C} \gamma$ receptor
95\% confidence interval $(\mathrm{CI}), 0.89-5.66 ; \mathrm{P}=0.09]$. Univariate analysis revealed increased tumor shrinkage in patients with the $\mathrm{K} / \mathrm{K}$ polymorphism of EGFR compared with the other polymorphisms (mean \pm standard deviation, $-55.3 \pm 28.4$ vs. $-39.6 \pm 40.8 \%$; $\mathrm{P}=0.04$ ). Subsequent multivariate analysis confirmed that the $\mathrm{K} / \mathrm{K}$ polymorphism of EGFR predicted greater tumor shrinkage (multiple linear regression analysis estimate, $-19.3 ; 95 \% \mathrm{CI},-35.5$ to $3.0 ; \mathrm{P}=0.02$ ), with the tendency toward a preferable response in patients with $<36$ CA EGFR gene repeats (estimate, $-16.9 ; 95 \% \mathrm{CI}$; -34.4 to 0.6 ; $\mathrm{P}=0.06$ ). However, other polymorphisms and clinical variables did not predict tumor shrinkage. In conclusion, the present study demonstrated that polymorphisms of EGFR, Fc $\gamma$ R2A and $\mathrm{Fc} \gamma \mathrm{R} 3 \mathrm{~A}$ may differentiate the patients that obtain the maximum benefit from cetuximab treatment.

\section{Introduction}

Epidermal growth factor receptor (EGFR) is a receptor tyrosine kinase found on the cell surface that is often upregulated in tumor cells (1). EGFR plays a pivotal role in cell proliferation by activating downstream signaling pathways (2). Cetuximab, a chimeric immunoglobulin G1 monoclonal antibody against EGFR, blocks the function of EGFR by competitively antagonizing and/or internalizing the receptor $(3,4)$. Clinical studies have demonstrated its efficacy in the treatment of a number of different types of cancer, including advanced and metastatic colorectal cancer (mCRC) $(1,4)$.

Signaling from EGFR is relayed by a GTPase transducer protein named RAS, and RAS-associated mutations in tumor 
cells are associated with resistance to cetuximab treatment $(5,6)$. Therefore, the clinical use of cetuximab is limited to patients with RAS wild-type mCRC. In addition, RAS is not a sufficient biomarker for predicting tumor response, and disease control is observed in only half of patients with KRAS wild-type mCRC subjected to monotherapy as first- or later-line treatment $(7,8)$. Therefore, additional predictors of tumor response to cetuximab are required in order to avoid poor treatment efficacy with unnecessary adverse reactions.

Antibody-dependent, cell-mediated cytotoxicity (ADCC) is proposed as a distinct mechanism of antitumor activity by cetuximab, and thus has gathered attention as a potential predictor of treatment efficacy and/or safety $(9,10)$. Cetuximab has an antigen-binding and crystalline fragment ( $\mathrm{Fc}$ fragment) in its structure $(9,11)$ allowing it to bind to both the tumor antigen (EGFR) and fragment $\mathrm{C} \gamma$ receptor $(\mathrm{Fc} \gamma \mathrm{R})$ located on immune cells, and to trigger ADCC (11). A histidine $(\mathrm{H}) / \operatorname{arginine}(\mathrm{R})$ polymorphism at position 131 on $\mathrm{Fc} \gamma \mathrm{R} 2 \mathrm{~A}$ and a valine $(\mathrm{V}) /$ phenylalanine $(\mathrm{F})$ polymorphism at position 158 on $\mathrm{Fc} \gamma \mathrm{R} 3 \mathrm{~A}$ are associated with different affinities for human IgG (12). According to the accumulating evidence, patients harboring $\mathrm{Fc} \gamma \mathrm{R} 2 \mathrm{~A}-131 \mathrm{H} / \mathrm{H}$ and $\mathrm{Fc} \gamma \mathrm{R} 3 \mathrm{~A}-158 \mathrm{~V} / \mathrm{V}$ mutations are expected to have stronger ADCC during monoclonal antibody therapies (13-15).

Furthermore, higher EGFR expression levels due to lower numbers of CA repeats in EGFR intron 1 may increase the response to cetuximab $(16,17)$. In addition, a substitution from $\mathrm{R}$ to lysine $(\mathrm{K})$ in codon 521 of the extracellular domain of EGFR could result in lower ligand binding affinity, downregulation of the target gene, and consequent favorable response to cetuximab treatment $(18,19)$. Despite these promising findings, clinical studies remain scarce, and interpretations of the results are conflicting due to several limiting factors. The present study thus investigated the association between gene polymorphisms in Fc $\gamma \mathrm{R} 2 \mathrm{~A}, \mathrm{Fc} \gamma \mathrm{R} 3 \mathrm{~A}$ and EGFR and the efficacy of first-line cetuximab and oxaliplatin treatment in patients with extended RAS/BRAF wild-type mCRC.

\section{Materials and methods}

Patients. The present study reviewed the clinical data of patients participating in one of two trials evaluating the efficacy of combination therapy with cetuximab and oxaliplatin-based chemotherapy as a first-line treatment (UMIN 000003253 and UMIN000007195) $(20,21)$. The patients were recruited, and the specimen was collected from 31 institutes in Japan between April 2010 and May 2011, and between February, 2012 and February, 2013. These institutes included Chiba Cancer Center (Chiba, Japan); Fukui-Ken Saiseikai Hospital (Fukui, Japan); Gifu University Hospital (Gifu, Japan); Hokkaido Cancer Center (Sapporo, Japan); Ishikawa Prefectural Central Hospital (Kanazawa, Japan); Japan Community Health Care Organization (JCHO) Osaka Hospital (Osaka, Japan); Kagawa University Hospital (Kita, Japan); Kanagawa Cancer Center (Yokohama, Japan); Kanazawa Medical University Hospital (Kahoku, Japan); Kansai Medical University Hospital (Hirakata, Japan); Kitakyushu General Hospital (Kitakyushu, Japan); Kobe Ekisaikai Hospital (Kobe, Japan); Kochi Medical School Hospital (Nankoku, Japan); Matsunami General Hospital (Hashima, Japan);
Nakadori General Hospital (Akita, Japan); National Hospital Organization Nagoya Medical Center (Nagoya, Japan); National Hospital Organization Osaka National Hospital (Osaka, Japan); Osaka City University Graduate School and Faculty of Medicine (Osaka, Japan); Osaka General Medical Center (Osaka, Japan); Osaka Rosai Hospital (Sakai, Japan); Osakakita Teishin Hospital (Osaka, Japan); Rinku General Medical Center (Izumisano, Japan); Sakai City Medical Center (Sakai, Japan); Sano Hospital (Kobe, Japan); Showa University Fujigaoka Hospital (Yokohama, Japan); Teikyo University Chiba Medical Center (Ichihara, Japan); Toyama Prefectural Central Hospital (Toyama, Japan); University of Occupational and Environmental Health (Kitakyushu, Japan); Toyonaka Municipal Hospital (Toyonaka, Japan); Yamaguchi University Hospital (Ube, Japan); and Yokoyama Hospital for Gastroenterological Diseases (Nagoya, Japan). The primary endpoint of these two trials was response rates (RRs) with confirmation, as evaluated by computed tomography at 4- to 8-weekly intervals. The RR in the present study was regarded as the clinically important primary endpoint as per the previous two clinical trials $(20,21)$. In total, 90 patients, with RAS/BRAF wild-type mCRC were identified, and the polymorphisms of these patients were analyzed. The mean age of the patients was $66.3 \pm 9.9$ years (standard deviation). The present study was approved by the Institutional Review Board of Yamaguchi University School of Medicine (approval number, H28-171) and performed in accordance with the Declaration of Helsinki. The requirement for informed consent was waived as the present study was a retrospective analysis of previously collected samples and data. The patients were given the opportunity to refuse the use of their samples in the present study, according to the ethics guidelines of the Institutional Review Board. Formalin-fixed, paraffin-embedded (FFPE) samples collected as part of the previous studies were used to analyze polymorphisms in Fc $\gamma$ R2A, Fc $\gamma$ R3A and EGFR for the present study. The data used in the present study was collected by Case Report Form for each clinical trial. No additional data/samples were collected for the present study.

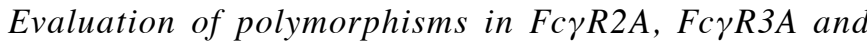
$E G F R$. DNA was extracted from the FFPE samples. Applying micro-dissection on $10 \mu \mathrm{m}$ sections, non-tumor tissues were dissected from the FFPE samples. DNA extraction was performed using a QIAamp DNA FFPE Tissue kit (Qiagen $\mathrm{GmbH}$ ) according to the manufacturer's protocol. The TaqMan technique was then used to determine Fc $\gamma$ R2A-H131R rs1801274, Fc $\gamma$ R3A-V158F rs396991 and EGFR-R521K rs2227983 polymorphisms using established primers (22), TaqMan SNP Genotyping Assays C_9077561_20, C_25815666_10 and C_16170352_20 (Applied Biosystems; Thermo Fisher Scientific, Inc.) and the TaqMan Genotyping Master Mix (Applied Biosystems; Thermo Fisher Scientific, Inc.). In brief, a 5- $\mu 1$ reaction solution, containing TaqMan Genotyping Master Mix (Applied Biosystems; Thermo Fisher Scientific, Inc.), Assay Mix, and 20-40 ng of genomic DNA diluted in $\mathrm{dH}_{2} \mathrm{O}$, was incubated in 384-well microtiter plates at $50^{\circ} \mathrm{C}$ for $2 \mathrm{~min}$ to degrade $\mathrm{dU}$-containing DNA, followed by incubation at $95^{\circ} \mathrm{C}$ for $10 \mathrm{~min}$ (denaturation), followed by 40 cycles of $15 \mathrm{sec}$ at $95^{\circ} \mathrm{C}$ and $1 \mathrm{~min}$ of annealing and extension at $60^{\circ} \mathrm{C}$. The ABI Prism 7900HT (Applied Biosystems; 
Table I. Clinical characteristics and frequencies of polymorphisms.

\begin{tabular}{lc} 
Variables & Number of patients \\
\hline Sex & \\
Female & $34(37.8)$ \\
Male & $56(62.2)$ \\
ECOG-PS & \\
0 & $79(87.8)$ \\
1 & $11(12.2)$ \\
Treatment & \\
FOLFOX & $37(41.1)$ \\
CapeOX & $53(58.9)$ \\
Primary tumor site & \\
Colon & $51(56.7)$ \\
Rectum & $39(43.3)$ \\
Fc $\gamma$ R2A (H131R) & \\
H/H & $55(61.1)$ \\
H/R & $31(34.4)$ \\
R/R & $3(3.3)$ \\
Not determined & $1(1.1)$ \\
Fc $\gamma$ R3A (V158F) & \\
V/V & \\
V/F & $33(36.7)$ \\
F/F & $39(43.3)$ \\
EGFR (R521K) & $18(20.0)$ \\
K/K & \\
K/R & $35(38.9)$ \\
R/R & \\
EGFR (CA repeat) & \\
$<36$ & $(47.8)$ \\
$\geq 36$ & \\
\hline
\end{tabular}

ECOG-PS, Eastern Cooperative Oncology Group Performance Status; FOLFOX, FOLFOX + cetuximab treatment, CapeOX, CapeOX + cetuximab treatment; EGFR, epidermal growth factor receptor; $\mathrm{Fc} \gamma \mathrm{R}$, fragment $\mathrm{C} \gamma$ receptor; $\mathrm{H}$, histidine; $\mathrm{V}$, valine; $\mathrm{K}$, lysine; $\mathrm{R}$, arginine; $\mathrm{F}$, phenylalanine.

Thermo Fisher Scientific, Inc.) was used for end-point reading of the fluorescence generated during PCR amplification. EGFR CA Repeats in Intron 1 Genotyping was determined via direct sequencing, as previously described $(23,24)$.

Statistical analysis. The primary endpoint was RR and the secondary endpoint was the maximum change in tumor diameter from baseline, calculated using the formula (tumor diameter at evaluation-tumor diameter at baseline)/tumor diameter at baseline x100, whereby negative numbers indicate tumor shrinkage during the treatment and positive numbers indicate tumor enlargement.

The Eastern Cooperative Oncology Group Performance Status (ECOG-PS) (25), combined chemotherapy, patient sex and primary tumor sites were used as variables that may potentially affect treatment efficacy. The detailed information regarding tumor location in the colon (i.e. left- or right-side colon) was not collected in the previous clinical trials and was therefore unavailable in the present study. In order to analyze the association between the tumor response and variables, $\chi^{2}$ test was performed, followed by logistic regression analysis. For the association between tumor shrinkage and variables, Welch's t-test was performed, followed by multiple linear regression analysis. $\mathrm{P}<0.05$ was considered to indicate a statistically significant difference. Statistical analyses and graph depiction were performed using Microsoft Excel (version 2013; Microsoft Corporation) and KaleidaGraph 4.5 (version 4.5; Synergy Software). The final figures were created using Photoshop CS2 (Adobe Systems).

\section{Results}

Frequency of polymorphisms and mutation status. $\mathrm{H} / \mathrm{H}$ in Fc $\gamma \mathrm{R} 2 \mathrm{~A}$ was the most frequent genetic polymorphism observed in the present study $(61.1 \%)$, while V/V in Fc $\gamma \mathrm{R} 3 \mathrm{~A}$ was observed in only 12 patients $(13.3 \%$ ) (Table I). K/K in EGFR was observed in 33 patients $(36.7 \%)$, while $27.8 \%$ of the patients had $<36 \mathrm{CA}$ repeats in EGFR. In one patient, $\mathrm{Fc} \gamma \mathrm{R} 2 \mathrm{~A}$ polymorphisms could not be determined due to DNA fragmentation, and their data concerning Fc $\gamma \mathrm{R} 2 \mathrm{~A}$ were excluded from further analysis.

In terms of the mutation status, one of the two clinical trials only recruited patients with KRAS wild-type CRC. Therefore, the total rate of RAS/BRAF mutations could not be assessed in this retrospective study.

$R R$. The univariate analysis demonstrated no significant difference in RR between patients with and without the tested polymorphisms (Table II). Therefore, the odds ratio for tumor response was estimated using all listed variables (Fig. 1). The patients with an $\mathrm{H} / \mathrm{H}$ polymorphism in $\mathrm{Fc} \gamma \mathrm{R} 2 \mathrm{~A}$ (vs. non- $\mathrm{H} / \mathrm{H}$ polymorphism) had an odds ratio of 2.25 , although this was not statistically significant $(\mathrm{P}=0.09)$.

Maximum change in tumor diameter from baseline. The maximum change in tumor diameter from baseline was used as a secondary endpoint in the present study to investigate the influence of gene polymorphisms. As the present study included patients who had received cetuximab in addition to conventional cytotoxic chemotherapy, the maximum change in tumor diameter from baseline (continuous scale) was considered to be a more sensitive endpoint for measuring the association between polymorphisms and treatment efficacy. As the tumor diameter information was unavailable for one patient, the analyses were performed using the data of 89 patients.

Notably, patients with a $\mathrm{K} / \mathrm{K}$ polymorphism in the EGFR gene exhibited greater tumor shrinkage compared with the patients with $K / R$ or $R / R$ in the EGFR gene ( $P=0.04$; Fig. 2). The multivariate analysis demonstrated a significant association between the $\mathrm{K} / \mathrm{K}$ polymorphism in EGFR and tumor shrinkage [multiple linear regression analysis estimate, -19.3; $95 \%$ confidence interval $(\mathrm{CI}),-35.5$ to $3.0 ; \mathrm{P}=0.02]$ (Table III). Patients with $<36 \mathrm{CA}$ repeats in the EGFR gene exhibited a tendency toward a better tumor response (estimate, $-16.9 ; 95 \%$ 
Table II. Univariate analysis for response rate.

\begin{tabular}{|c|c|c|c|c|}
\hline Variables & $\mathrm{CR}$ or $\mathrm{PR}, \mathrm{n}$ & $\mathrm{RR}, \%$ & $\chi^{2}$ & P-value \\
\hline Sex & & & 0.48 & 0.49 \\
\hline Female & 20 & 58.8 & & \\
\hline Male & 37 & 66.1 & & \\
\hline ECOG-PS & & & 0.42 & 0.52 \\
\hline 0 & 51 & 64.6 & & \\
\hline 1 & 6 & 54.5 & & \\
\hline Treatment & & & 0.06 & 0.80 \\
\hline FOLFOX & 24 & 64.9 & & \\
\hline CapeOX & 33 & 62.3 & & \\
\hline Primary tumor site & & & 0.33 & 0.57 \\
\hline Colon & 31 & 60.8 & & \\
\hline Rectum & 26 & 66.7 & & \\
\hline $\mathrm{Fc} \gamma \mathrm{R} 2 \mathrm{~A}(\mathrm{H} 131 \mathrm{R})$ & & & 2.95 & 0.09 \\
\hline $\mathrm{H} / \mathrm{H}$ & 39 & 70.9 & & \\
\hline Non-H/H & 18 & 52.9 & & \\
\hline $\mathrm{Fc} \gamma \mathrm{R} 3 \mathrm{~A}(\mathrm{~V} 158 \mathrm{~F})$ & & & 0.07 & 0.80 \\
\hline $\mathrm{V} / \mathrm{V}$ & 8 & 66.7 & & \\
\hline Non-V/V & 49 & 62.8 & & \\
\hline EGFR (R521K) & & & 0.25 & 0.62 \\
\hline $\mathrm{K} / \mathrm{K}$ & 22 & 66.7 & & \\
\hline Non-K/K & 35 & 61.4 & & \\
\hline EGFR (CA repeat) & & & 0.32 & 0.57 \\
\hline$<36$ & 17 & 68.0 & & \\
\hline$\geq 36$ & 40 & 61.5 & & \\
\hline
\end{tabular}

CR, complete response; PR, partial response; RR, response rate; ECOG-PS, Eastern Cooperative Oncology Group Performance Status; FOLFOX, FOLFOX + cetuximab treatment, CapeOX, CapeOX + cetuximab treatment; EGFR, epidermal growth factor; Fc $\gamma \mathrm{R}$, fragment $\mathrm{C} \gamma$ receptor; $\mathrm{H}$, histidine; $\mathrm{V}$, valine; $\mathrm{K}$, lysine; $\mathrm{R}$, arginine; $\mathrm{F}$, phenylalanine.

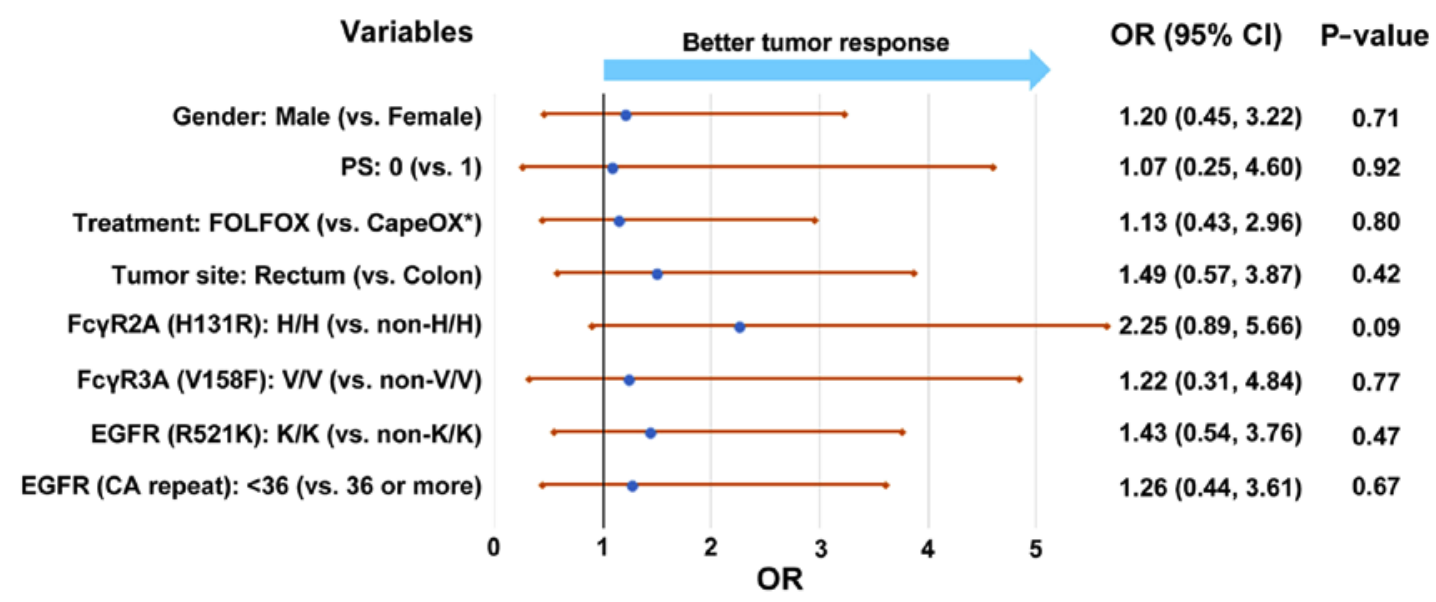

Figure 1. OR for response rate. A value of $>1$ suggested that the patient groups were more likely to exhibit a complete or partial response compared with the counterparts. No significant difference was observed. OR, odds ratio; CI, confidence interval; EGFR, epidermal growth factor receptor; Fc $\gamma$ R, fragment $\mathrm{C} \gamma$ receptor; $\mathrm{H}$, histidine; $\mathrm{V}$, valine; $\mathrm{K}$, lysine; PS, performance status.

CI, -34.4 to $0.6 ; \mathrm{P}=0.06)$. Tumor size change at individual level was also compared between patients with and without the K/K EGFR polymorphism (Fig. 3), and was consistent with the univariate analysis in that those with the polymorphism exhibited greater tumor shrinkage than those without the gene change. However, it should be noted that there were certain 


\begin{tabular}{|c|c|c|c|c|}
\hline Variables & $\mathbf{n}$ & Mean $(95 \% \mathrm{Cl})$ & & P-value \\
\hline $\begin{array}{l}\text { Gender } \\
\text { Male } \\
\text { Female }\end{array}$ & $\begin{array}{l}55 \\
34\end{array}$ & $\begin{array}{l}-44.7(-54.8,-34.6) \\
-46.5(-59.7,-33.3)\end{array}$ & . & 0.83 \\
\hline \multicolumn{5}{|l|}{ PS } \\
\hline $\mathbf{0}$ & 78 & $-46.4(-54.6,-38.2)$ & $\cdots+100$ & 0.59 \\
\hline 1 & 11 & $-38.6(-68.7,-8.4)$ & $\square-\cdots$ & \\
\hline \multicolumn{5}{|l|}{ Treatment } \\
\hline FOLFOX & 36 & $-51.1(-64.3,-37.8)$ & $\cdots-1$ & 0.25 \\
\hline CapeOX & 53 & $-41.5(-51.4,-31.6)$ & $\cdots+100$ & \\
\hline \multicolumn{5}{|c|}{ Primary tumor site } \\
\hline Colon & 50 & $-43.2(-53.4,-32.9)$ & $\circ \circ \circ$ & 0.53 \\
\hline Rectum & 39 & $-48.3(-60.9,-35.6)$ & 0 & \\
\hline \multicolumn{5}{|c|}{ FcyR2A (H131R) } \\
\hline $\mathrm{H} / \mathrm{H}$ & 54 & $-48.7(-58.6,-38.9)$ & $\circ \circ$ & 0.43 \\
\hline Non-H/H & 34 & $-42.2(-55.5,-28.9)$ & n......-1 & \\
\hline \multicolumn{5}{|c|}{ FcyR3A (V158F) } \\
\hline V/N & 12 & $-47.0(-73.8,-20.2)$ & $\circ$ & 0.89 \\
\hline Non-V/V & 77 & $-45.1(-53.5,-36.8)$ & $\cdots-1 \circ 0$ & \\
\hline \multicolumn{5}{|l|}{ EGFR (R521K) } \\
\hline $\mathbf{K} / \mathbf{K}$ & 33 & $-55.3(-65.3,-45.2)$ & $\circ$ & 0.04 \\
\hline Non-K/K & 56 & $-39.6(-50.5,-28.7)$ & - 0 & \\
\hline \multicolumn{5}{|c|}{ EGFR (CA repeat) } \\
\hline$<36$ & 24 & $-54.0(-67.1,-41.0)$ & $\cdots-1$ & 0.14 \\
\hline 36 or more & 65 & $-43.1(-51.9,-32.5)$ & - & \\
\hline
\end{tabular}

Figure 2. Degree of tumor size change and univariate analysis. Maximum changes in tumor diameter from baseline is presented according to the variables. Negative numbers indicate tumor shrinkage, while positive numbers indicate tumor enlargement. Enhanced tumor shrinkage was observed among the patients with $\mathrm{K} / \mathrm{K}$ polymorphisms in EGFR (R521K) compared with the patients with no K/K polymorphism. EGFR, epidermal growth factor receptor; CI, confidence interval; Fc $\gamma \mathrm{R}$, fragment $\mathrm{C} \gamma$ receptor; $\mathrm{H}$, histidine; V, valine; $\mathrm{K}$, lysine; R, arginine; F, phenylalanine; PS, performance status.

patients that exhibited sufficient tumor shrinkage among patients with $\mathrm{K} / \mathrm{R}$ or $\mathrm{R} / \mathrm{R}$ in the EGFR gene.

\section{Discussion}

Previous studies investigating the association between polymorphisms of $\mathrm{Fc} \gamma \mathrm{R}$ and cetuximab treatment had several limitations. First, only $\mathrm{mCRC}$ harboring KRAS exon2 mutations were excluded $(10,26,27)$, and thus the influence of other mutations, such as extended RAS and BRAF, could not be ruled out. Secondly, a number of studies analyzed the combined data of patients with substantially divergent backgrounds, including line of the treatment, backbone of the chemotherapy (oxaliplatin, irinotecan or monotherapy) and even monotherapy $(9,28)$. As discussed by Inoue et al $(22)$, the deteriorated systemic and local immune systems in heavily treated patients could possibly exert only limited antitumor activity mediated by ADCC; analyzing these data without considering these factors may have led to conflicting results. In contrast, the uniquely valuable characteristic features of the present study are the exclusion of patients with mCRC that exhibited BRAF or extended RAS mutations, the inclusion of only first-line treatment regimens, and limiting the backbone treatment to oxaliplatin and fluoropyrimidines.

Under these conditions, two results were revealed: i) A clear association between the K/K polymorphism of EGFR and maximum tumor shrinkage from baseline; and ii) a tendency toward greater efficacy in tumors carrying the $\mathrm{H} / \mathrm{H}$ polymorphism of $F c \gamma R 2 A$. The former result is partly consistent with previous suggestions of an improved prognosis in patients with the $\mathrm{K} / \mathrm{K}$ polymorphism $(18,19)$, including the observation that tumors harboring $\mathrm{K} / \mathrm{K}$ or $\mathrm{K} / \mathrm{R}$ exhibited favorable tumor characteristics and a higher RR to cetuximab combined chemotherapy in 112 patients with KRAS wild-type colorectal carcinoma (18). Such a result could reflect attenuated EGFR signaling and the higher sensitivity to signaling blockade by cetuximab in patients with the R521K polymorphism (18). Unlike colorectal cancer, expression of the K-allele in head and neck cancers has been associated with shorter progression free survival (PFS) and resistance to cetuximab, with stronger treatment required to induce $\mathrm{K}$-alleles in ADCC cells in vitro, due to lower affinity (29). Although no clear explanation has yet emerged for these inconsistent observations, differing dependencies on EGFR signaling among different tumor types and different degrees of required antibody affinity for signal inhibition by cetuximab are both possible underlying mechanisms (29). Further studies are required in order to elucidate these aspects.

In contrast to the tumor shrinkage effects, polymorphisms of Fc $\gamma$ R2A, Fc $\gamma$ R3A and EGFR had no statistically significant association with tumor response.. Nevertheless, the multivariate analysis demonstrated a tendency for an improved tumor response in $\mathrm{H} / \mathrm{H}$ tumors compared with non- $\mathrm{H} / \mathrm{H}$ tumors. Specifically, a H/R polymorphism at position 131 on $\mathrm{Fc} \gamma \mathrm{R} 2 \mathrm{~A}$ was associated with enhanced affinities for human $\mathrm{IgG}$, and patients harboring $\mathrm{Fc} \gamma \mathrm{R} 2 \mathrm{~A}-131 \mathrm{H} / \mathrm{H}$ mutations were predicted to have stronger ADCC (13-15). A study using the data and samples from patients receiving cetuximab monotherapy for colorectal cancer demonstrated a significant association between efficacy of late-line cetuximab monotherapy and an $\mathrm{H} / \mathrm{H}$ polymorphism in Fc $\gamma \mathrm{R} 2 \mathrm{~A}$ (10). The present study therefore investigated 
Table III. Multiple linear regression analysis for maximum tumor change from baseline.

\begin{tabular}{lccr}
\hline Variables & Groups & Estimate (SE) & $95 \%$ CI \\
\hline Sex & Male vs. female & $1.6(8.7)$ & $-15.4,18.6$ \\
ECOG-PS & 1 vs. 0 & $-0.8(12.6)$ & $-25.7,24.2$ \\
Treatment & FOLFOX vs. CapeOX & $-10(8.2)$ & $-26.4,6.3$ \\
Primary tumor site & Colon vs. rectum & $6.5(8.1)$ & $-9.7,22.6$ \\
Fc $\gamma$ R2A (H131R) & H/H vs. non-H/H & $-6.2(8)$ & $-22.1,9.7$ \\
Fc $\gamma$ R3A (V158F) & V/V vs. non-V/V & $-6.1(11.6)$ & $-29.2,17$ \\
EGFR (R521K) & K/K vs. non-K/K & $-19.3(8.2)$ & $-35.5,-3.0$ \\
EGFR (CA repeat) & $<36$ vs. $\geq 36$ & $-16.9(8.8)$ & $-34.4,0.6$ \\
\hline
\end{tabular}

ECOG-PS, Eastern Cooperative Oncology Group Performance Status; FOLFOX,FOLFOX + cetuximab treatment; CapeOX, CapeOX + cetuximab treatment; SE, standard error; CI, confidence interval; EGFR, epidermal growth factor; Fc $\gamma \mathrm{R}$, fragment $\mathrm{C} \gamma$ receptor; $\mathrm{H}$, histidine; V, valine; K, lysine; R, arginine; F, phenylalanine.

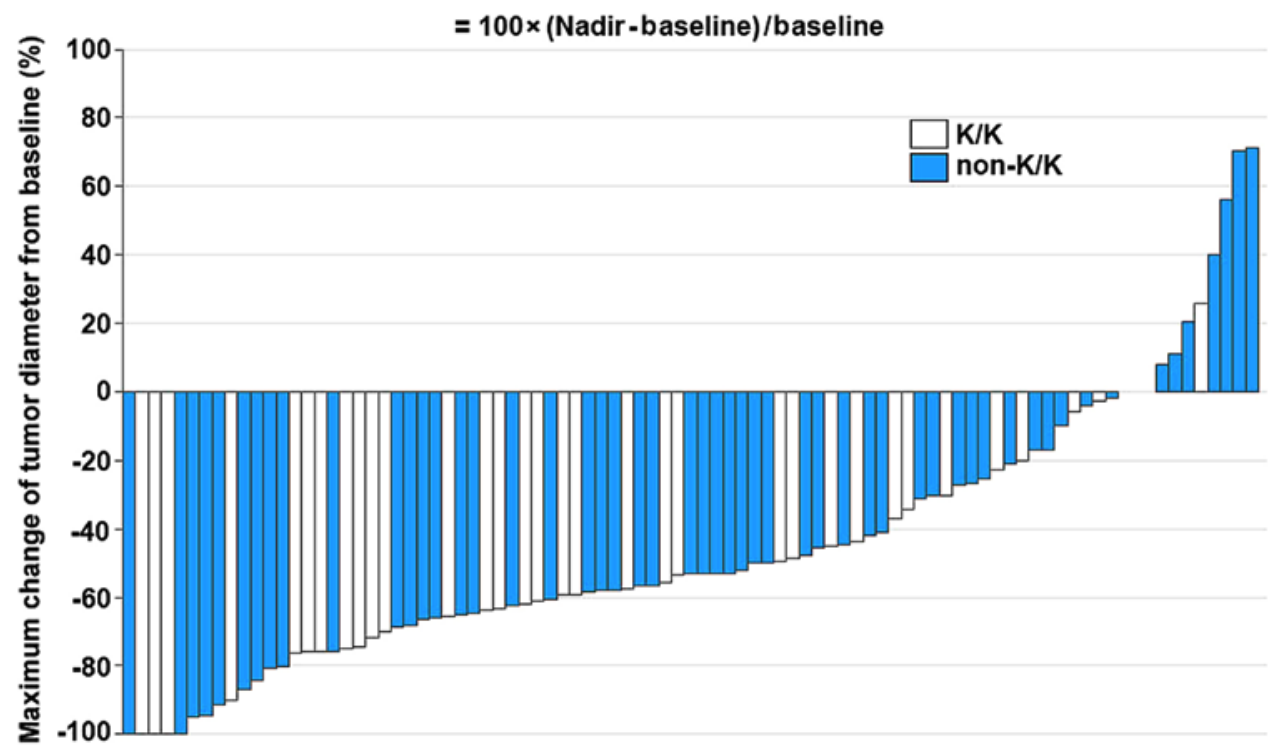

Figure 3. Waterfall plots presenting the change in tumor size in 89 patients. The individual data for tumor size is presented in white for the patients with a $\mathrm{K} / \mathrm{K}$ polymorphism in EGFR (R521K) and in blue for the patients with no K/K polymorphism. EGFR, epidermal growth factor receptor; K, lysine; R, arginine.

whether combination oxaliplatin-based chemotherapy could obscure the association between tested polymorphisms and RR, as cytotoxic-doublet treatment is generally effective in $50 \%$ of patients with mCRC. In addition, a number of the patients recruited in the clinical trials assessed during the present study received a hepatectomy with curative intent, which would significantly influence PFS and overall survival.

The incidence of $\mathrm{H} / \mathrm{H}$ polymorphisms in $\mathrm{Fc} \gamma \mathrm{R} 2 \mathrm{~A}$ and $\mathrm{V} / \mathrm{V}$ in $\mathrm{Fc} \gamma \mathrm{R} 3 \mathrm{~A}$ in the present study was 61 and $13 \%$, respectively. A previous study demonstrated that the incidence of $\mathrm{H} / \mathrm{H}$ in Fc $\gamma$ R2A was higher among Japanese patients than patients from Europe and the USA (9), and 61\% in the present study is consistent with this and other previous study $(9,22)$. In contrast, a 4-9\% incidence rate of the $\mathrm{V} / \mathrm{V}$ polymorphism in $\mathrm{Fc} \gamma \mathrm{R} 3 \mathrm{~A}$ has been demonstrated $(9,21)$, suggesting a slightly higher incidence of $\mathrm{V} / \mathrm{V}$ in $\mathrm{Fc} \gamma \mathrm{R} 3 \mathrm{~A}$ in the present study; however, the frequency of polymorphisms of certain gene differs within Japan (30), and such variability could account for the small differences in incidence between previous studies and the present study. Although external validation was not performed in the present study, the similarities in polymorphism frequencies and the use of an established primers (22) and methods described above ensure reliability in the laboratory evaluations.

A limitation of the present study was the potential effect of polymorphisms on the treatment efficacy of cytotoxic agents, such as oxaliplatin and fluoropyrimidines. Although this is an unlikely outcome, the issue may be overcome by setting FOLFOX/CapeOX alone as a control arm and demonstrating the lack of polymorphism effects in this control group. Analyzing the data of patients receiving cetuximab monotherapy would be an alternative resolution, although cetuximab monotherapy is rarely utilized as a first- or second-line treatment. Instead, monotherapy is often used only in patients with deteriorated general status (31) or as a later treatment option, and in both these cases the immune system and ADCC may not function as expected. Another limitation was the lack of information regarding the sidedness of the primary tumors. As sidedness of the primary tumor is recognized as being significantly 
associated with the efficacy of anti-EGFR therapy (32), adding sidedness to the other clinical data may further clarify the impact of polymorphisms.

In conclusion, the present study provides preliminary evidence suggesting an association between treatment efficacy and polymorphisms in the EGFR gene in patients with RAS/BRAF wild-type mCRC. Individuals harboring the $\mathrm{K} / \mathrm{K}$ polymorphism in EGFR demonstrated significantly greater tumor shrinkage during treatment than those with the non-K/K polymorphism. Further studies with an appropriate control arm and endpoints of clinical importance are necessary.

\section{Acknowledgements}

Not applicable.

\section{Funding}

The present study was supported, in part, by the non-profit organization Epidemiological \& Clinical Research Organization (ECRIN).

\section{Availability of data and materials}

The datasets used and/or analyzed during the present study are available from the corresponding author upon reasonable request.

\section{Authors' contributions}

HMa, SH, SI, KO, JS, HMi and NN conceptualized the study, curated the data and wrote the original draft of the manuscript. KO, RT, NO, YS, TY, YN, NS, HN, JS, HMa and NN performed the analyses, investigations and all methodology. SH, KO, RT, NO, YS, TY, YN, JS, HMi and NN performed the project administration. HMa, SH, JS and HMi wrote, reviewed and edited the manuscript. HMi and NN supervised the investigations. All authors read and approved the final manuscript.

\section{Ethics approval and consent to participate}

The present study was approved by the Institutional Review Board of Yamaguchi University School of Medicine. The requirement for informed consent was waived as the samples had been collected as part of previous clinical trials. The patients were given the opportunity to refuse that their samples be used in the present study.

\section{Patient consent for publication}

Not applicable.

\section{Competing interests}

JS has received honoraria from Tsumura, Chugai Pharmaceutical and consulting fees from Takeda Pharmaceutical. KO has received honoraria (lecture and/or manuscript fees) from Takeda Pharmaceutical Company Ltd., Bristol-Myers Squibb Company Ltd., Ono Pharmaceutical Co. Ltd. and Chugai Pharmaceutical Co. Ltd. SH and HN received research funding from NEC Corporation, Toyo Kohan Corporation and Merck Serono Co., Ltd. HMis has received honoraria from Chugai Pharmaceutical, Takeda Pharmaceutical and Taiho Pharmaceutical. NN has received honoraria from Takeda Pharmaceutical Company Ltd. The other co-authors declare that they have no competing interests.

\section{References}

1. Kim ES, Khuri FR and Herbst RS: Epidermal growth factor receptor biology (IMC-C225). Curr Opin Oncol 13: 506-513, 2001.

2. Woodburn JR: The epidermal growth factor receptor and its inhibition in cancer therapy. Pharmacol Ther 82: 241-250, 1999.

3. Galizia G, Lieto E, De Vita F, Orditura M, Castellano P, Troiani T, Imperatore V and Ciardiello F: Cetuximab, a chimeric human mouse anti-epidermal growth factor receptor monoclonal antibody, in the treatment of human colorectal cancer. Oncogene 26: 3654-3660, 2007.

4. Harris M: Monoclonal antibodies as therapeutic agents for cancer. Lancet Oncol 5: 292-302, 2004.

5. Chetty R and Govender D: Gene of the month: KRAS. J Clin Pathol 66: 548-550, 2013.

6. Zhao B, Wang L, Qiu H, Zhang M, Sun L, Peng P, Yu Q and Yuan X: Mechanisms of resistance to anti-EGFR therapy in colorectal cancer. Oncotarget 8: 3980-4000, 2017.

7. Karapetis CS, Khambata-Ford S, Jonker DJ, O'Callaghan CJ, Tu D, Tebbutt NC, Simes RJ, Chalchal H, Shapiro JD, Robitaille S, et al: K-ras mutations and benefit from cetuximab in advanced colorectal cancer. N Engl J Med 359: 1757-1765, 2008.

8. Pessino A, Artale S, Sciallero S, Guglielmi A, Fornarini G, Andreotti IC, Mammoliti S, Comandini D, Caprioni F, Bennicelli E, et al: First-line single-agent cetuximab in patients with advanced colorectal cancer. Ann Oncol 19: 711-716, 2008.

9. Geva R, Vecchione L, Kalogeras KT, Jensen BV, Lenz HJ, Yoshino T, Paez D, Montagut C, Souglakos J, Cappuzzo F, et al: FCGR polymorphisms and cetuximab efficacy in chemorefractory metastatic colorectal cancer: An international consortium study. Gut 64: 921-928, 2015.

10. Liu G, Tu D, Lewis M, Cheng D, Sullivan LA, Chen Z, Morgen E, Simes J, Price TJ, Tebbutt NC, et al: $\mathrm{Fc}-\gamma$ receptor polymorphisms, cetuximab therapy, and survival in the NCIC CTG CO.17 trial of colorectal cancer. Clin Cancer Res 22: 2435-2444, 2016.

11. Weiner LM, Surana R and Wang S: Monoclonal antibodies: Versatile platforms for cancer immunotherapy. Nat Rev Immunol 10: 317-327, 2010.

12. van Sorge NM, van der Pol WL and van de Winkel JG: FcgammaR polymorphisms: Implications for function, disease susceptibility and immunotherapy. Tissue Antigens 61: 189-202, 2003.

13. Koene HR, Kleijer M, Algra J, Roos D, von dem Borne AE and de Haas M: Fc gammaRIIIa-158V/F polymorphism influences the binding of IgG by natural killer cell $\mathrm{Fc}$ gammaRIIIa, independently of the $\mathrm{Fc}$ gammaRIIIa-48L/R/H phenotype. Blood 90: 1109-1114, 1997.

14. Dall'Ozzo S, Tartas S, Paintaud G, Cartron G, Colombat P, Bardos P, Watier $\mathrm{H}$ and Thibault G: Rituximab-dependent cytotoxicity by natural killer cells: Influence of FCGR3A polymorphism on the concentration-effect relationship. Cancer Res 64: 4664-4669, 2004

15. Binstadt BA, Geha RS and Bonilla FA: IgG Fc receptor polymorphisms in human disease: Implications for intravenous immunoglobulin therapy. J Allergy Clin Immunol 111: 697-703, 2003.

16. Amador ML, Oppenheimer D, Perea S, Maitra A, Cusatis G, Iacobuzio-Donahue C, Baker SD, Ashfaq R, Takimoto C, Forastiere A and Hidalgo M: An epidermal growth factor receptor intron 1 polymorphism mediates response to epidermal growth factor receptor inhibitors. Cancer Res 64: 9139-9143, 2004.

17. Buerger H, Gebhardt F, Schmidt H, Beckmann A, Hutmacher K, Simon R, Lelle R, Boecker W and Brandt B: Length and loss of heterozygosity of an intron 1 polymorphic sequence of egfr is related to cytogenetic alterations and epithelial growth factor receptor expression. Cancer Res 60: 854-857, 2000.

18. Hsieh YY, Tzeng CH, Chen MH, Chen PM and Wang WS: Epidermal growth factor receptor R521K polymorphism shows favorable outcomes in KRAS wild-type colorectal cancer patients treated with cetuximab-based chemotherapy. Cancer Sci 103: 791-796, 2012. 
19. Gonçalves A, Esteyries S, Taylor-Smedra B, Lagarde A, Ayadi M, Monges G, Bertucci F, Esterni B, Delpero JR, Turrini O, et al: A polymorphism of EGFR extracellular domain is associated with progression free-survival in metastatic colorectal cancer patients receiving cetuximab-based treatment. BMC Cancer 8: 169, 2008

20. Soda H, Maeda H, Hasegawa J, Takahashi T, Hazama S, Fukunaga M, Kono E, Kotaka M, Sakamoto J, Nagata N, et al: Multicenter phase II study of FOLFOX or biweekly XELOX and Erbitux (cetuximab) as first-line therapy in patients with wild-type KRAS/BRAF metastatic colorectal cancer: The FLEET study. BMC Cancer 15: 695, 2015.

21. Hazama S, Maeda H, Iwamoto S, Kim HM, Takemoto H, Kobayashi K, Sakamoto J, Nagata N, Oba K and Mishima H: A phase II study of XELOX and cetuximab as first-line therapy in patients with KRAS wild type metastatic colorectal cancer (FLEET2 Study). Clin Colorectal Cancer 15: 329-336, 2016.

22. Inoue $\mathrm{Y}$, Hazama S, Iwamoto S, Miyake Y, Matsuda C, Tsunedomi R, Okayama N, Hinoda Y, Yamasaki T, Suehiro Y, et al: Fc $\gamma \mathrm{R}$ and EGFR polymorphisms as predictive markers of cetuximab efficacy in metastatic colorectal cancer Mol Diagn Ther 18: 541-548, 2014.

23. Okayama N, Nishioka M, Hazama S, Sakai K, Suehiro Y, Maekawa M, Sakamoto J, Iwamoto S, Kato T, Mishima H, et al: The importance of evaluation of DNA amplificability in KRAS mutation testing with dideoxy sequencing using formalin-fixed and paraffin-embedded colorectal cancer tissues. Jpn J Clin Oncol 41: 165-171, 2011.

24. Maruta Y, Okayama N, Hiura M, Suehiro Y, Hirai H and Hinoda Y: Determination of ancestral allele for possible human cancerassociated polymorphisms. Cancer Genet Cytogenet 180: 24-29, 2008.

25. Oken MM, Creech RH, Tormey DC, Horton J, Davis TE, McFadden ET and Carbone PP: Toxicity and response criteria of the Eastern Cooperative Oncology Group. Am J Clin Oncol 5: 649-655, 1982

26. Pander J, Gelderblom H, Antonini NF, Tol J, van Krieken JH, van der Straaten T, Punt CJ and Guchelaar HJ: Correlation of FCGR3A and EGFR germline polymorphisms with the efficacy of cetuximab in KRAS wild-type metastatic colorectal cancer. Eur J Cancer 46: 1829-1834, 2010
27. Kjersem JB, Skovlund E, Ikdahl T, Guren T, Kersten C, Dalsgaard AM, Yilmaz MK, Fokstuen T, Tveit KM and Kure EH: FCGR2A and FCGR3A polymorphisms and clinical outcome in metastatic colorectal cancer patients treated with first-line 5-fluorouracil/folinic acid and oxaliplatin +/- cetuximab. BMC Cancer 14: 340, 2014.

28. Park SJ, Hong YS, Lee JL, Ryu MH, Chang HM, Kim KP, Ahn YC, Na YS, Jin DH, Yu CS, et al: Genetic polymorphisms of $\mathrm{Fc} \gamma \mathrm{RIIa}$ and $\mathrm{Fc} \gamma \mathrm{R}$ IIIa are not predictive of clinical outcomes after cetuximab plus irinotecan chemotherapy in patients with metastatic colorectal cancer. Oncology 82: 83-89, 2012.

29. Braig F, Kriegs M, Voigtlaender M, Habel B, Grob T, Biskup K, Blanchard V, Sack M, Thalhammer A, Ben Batalla I, et al: Cetuximab Resistance in Head and Neck Cancer Is Mediated by EGFR-K521 Polymorphism. Cancer Res 77: 1188-1199, 2017.

30. Kobayashi M, Hazama S, Takahashi K, Oba K, Okayama N, Nishioka M, Hinoda Y, Oka M, Okamoto K, Maeda H, et al: Is there diversity among UGT1A1 polymorphism in Japan? World J Gastrointest Oncol 4: 170-175, 2012.

31. Van Cutsem E, Cervantes A, Adam R, Sobrero A, Van Krieken JH, Aderka D, Aranda Aguilar E, Bardelli A, Benson A, Bodoky G, et al: ESMO consensus guidelines for the management of patients with metastatic colorectal cancer. Ann Oncol 27: 1386-1422, 2016.

32. Tejpar S, Stintzing S, Ciardiello F, Tabernero J, Van Cutsem E, Beier F, Esser R, Lenz HJ and Heinemann V: Prognostic and predictive relevance of primary tumor location in patients with RAS wild-type metastatic colorectal cancer: Retrospective analyses of the CRYSTAL and FIRE-3 trials. JAMA Oncol 3: 194-201, 2017.

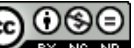

This work is licensed under a Creative Commons Attribution-NonCommercial-NoDerivatives 4.0 International (CC BY-NC-ND 4.0) License. 